BRAVILLIAN JOURNAL

www.bjournal.com.br
ISSN 0100-879X

Volume 43 (4) 268-380 April 2011

BIOMEDICAL SCIENCES

AND

CLINICAL INVESTIGATION

Braz J Med Biol Res, April 2011, Volume 44(4) 327-331

doi: 10.1590/S0100-879X2011007500021

\title{
Effect of neurotrophic factor, MDP, on rats' nerve regeneration
}

A.A. Fornazari, M.R. de Rezende, R. Mattar Jr., R.I. Taira, G.B. dos Santos and R.G. Paulos

The Brazilian Journal of Medical and Biological Research is partially financed by
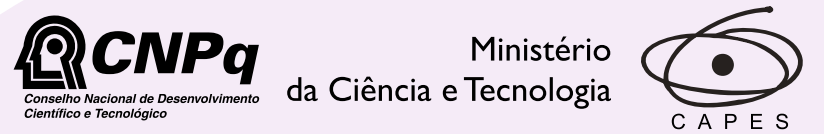

Ministério da Educação

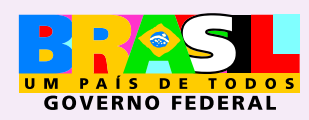

DTAPESP

Institutional Sponsors
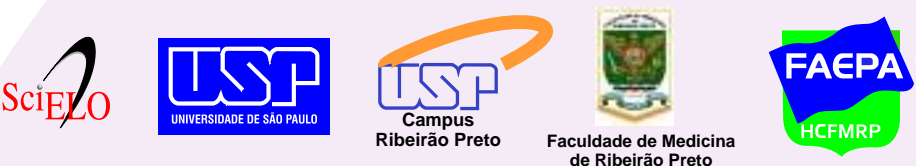


\title{
Effect of neurotrophic factor, MDP, on rats' nerve regeneration
}

\author{
A.A. Fornazari, M.R. de Rezende, R. Mattar Jr., R.I. Taira, \\ G.B. dos Santos and R.G. Paulos \\ Laboratório de Microcirurgia, Instituto de Ortopedia e Traumatologia, Hospital das Clínicas, \\ Universidade de São Paulo, São Paulo, SP, Brasil
}

\begin{abstract}
Our objective was to determine the immune-modulating effects of the neurotrophic factor $\mathrm{N}$-acetylmuramyl-L-alanyl-D-isoglutamine (MDP) on median nerve regeneration in rats. We used male Wistar rats (120-140 days of age, weighing 250-332 g) and compared the results of three different techniques of nerve repair: 1) epineural neurorrhaphy using sutures alone (group $S$ - 10 rats), 2) epineural neurorrhaphy using sutures plus fibrin tissue adhesive (FTA; group SF - 20 rats), and 3) sutures plus FTA, with MDP added to the FTA (group SFM - 20 rats). Functional assessments using the grasp test were performed weekly for 12 weeks to identify recovery of flexor muscle function in the fingers secondary to median nerve regeneration. Histological analysis was also utilized. The total number and diameter of myelinated fibers were determined in each proximal and distal nerve segment. Two indices, reported as percentage, were calculated from these parameters, namely, the regeneration index and the diameter change index. By the 8th week, superiority of group SFM over group $S$ became apparent in the grasping test $(P=0.005)$. By the 12th week, rats that had received MDP were superior in the grasping test compared to both group $S(P<$ $0.001)$ and group SF $(P=0.001)$. Moreover, group SF was better in the grasping test than group $S(P=0.014)$. However, no significant differences between groups were identified by histological analysis. In the present study, rats that had received MDP obtained better function, in the absence of any significant histological differences.
\end{abstract}

Key words: Grasping test; Neurotrophic factor; N-acetylmuramyl-L-alanyl-D-isoglutamine (MDP)

\section{Introduction}

When peripheral nerve is damaged it undergoes the degeneration process known as Wallerian degeneration. After nerve injury, a local inflammatory reaction occurs that involves macrophages. These cells, in turn, are responsible for many of the fundamental events that occur during the regenerative process. Macrophages play a crucial role in processes of Wallerian degeneration and subsequent regeneration of peripheral nerves (1). The use of neurotrophic factors has been of special interest to researchers investigating the best ways to stimulate nerve regeneration. $\mathrm{N}$-acetylmuramyl-Lalanyl-D-isoglutamine (MDP) is an immuno-adjuvant that promotes increased macrophage activity (2). When injected into the crushed sciatic nerve, MDP activates macrophages and promotes recovery of walking locomotion in rats (3). Some results indicate that locally applied MDP stimulates peripheral nerve regeneration in rats (4). Based on the feasibility of using fibrin glue mixed with the neurotrophic factor, we decided to study the immunomodulatory effect of
MDP on the process of peripheral nerve regeneration in a rat experimental model.

\section{Material and Methods}

\section{Animals}

Healthy male Wistar rats between 120 and 140 days of age and weighing 250-332 g were used, all without motor deficits. Rats were maintained in cages in groups of 5 . The cages were maintained in an acclimatized atmosphere under hygienic conditions, with food and water provided ad libitum. The study was approved by the Ethics Committee of Departamento de Ortopedia e Traumatologia, Hospital das Clínicas, Universidade de São Paulo (USP), Brazil, and was conducted according to the ethical guidelines for animal experimentation established by the Brazilian School of Animal Experimentation and by the Institute of Laboratory Animal Resources of the U.S. National Academy of Sciences.

Correspondence: A.A. Fornazari, Laboratório de Microcirurgia, Instituto de Ortopedia e Traumatologia, HC, USP, Rua Ovídio Pires de Campos, 333, 05403-010 São Paulo, SP, Brasil. Fax: +55-11-3067-7000. E-mail: adrienfornazari@ig.com.br

Received May 15, 2010. Accepted February 3, 2011. Available online February 25, 2011. Published April 11, 2011. 


\section{Groups}

Rats were anesthetized intraperitoneally (ip) with 30 to $40 \mathrm{mg} / \mathrm{kg}$ pentobarbital. An incision was made in the prepped and sterilized axillary area of the right upper extremity, and the median nerve was identified and dissected. The nerve was transected with micro-scissors $5 \mathrm{~mm}$ proximal to the medial epicondyle of the humerus and the lesion was immediately repaired always by the same surgeon.

Three groups of rats were used: group $S(N=10)$ was subjected to end-to-end epineural neurorrhaphy without tension, using four simple 10-0 mononylon sutures. Group SF $(N=20)$ was subjected to epineural neurorrhaphy without tension, using two simple sutures plus $0.10 \mathrm{~mL}$ fibrin tissue adhesive (FTA; Tissucol ${ }^{\circledR}$, Immuno AG, Austria). Group SFM $(N=20)$ was subjected to the same procedure as group $S F$, except that $0.1 \mathrm{~mL}$ neurotrophic factor MDP (Sigma Chemical Co., USA) was added to the FTA. MDP was maintained at $15^{\circ} \mathrm{C}$ and diluted in $1 \mathrm{~mL}$ saline to a 2-mM concentration. After adding an equal volume of $\mathrm{FTA}$, the final concentration of MDP was $1 \mathrm{mM}$. Surgery was performed under a light microscope at $12 \mathrm{X}$ magnification. After nerve repair, the surgical wound was closed with 3.0 mononylon sutures. Rats were excluded from analysis for the following reasons: perioperative or post-operative death of the animal, tissue loss in the injured area, autophagy or other significant mutilation in an animal, deep infection at the site of the lesion, and postoperative loss of more than $10 \%$ of preoperative weight. For the three groups, 50 rats were used. Six rats were excluded from the SFM group $(N=20)$. Two weeks postoperatively, 3 rats exhibited autophagy in the area of the surgical wound and significant motor alteration. During the 5th postoperative week, one rat died of an idiopathic cause. Two rats failed to exhibit any measurable flexor strength during the grasping test over the entire 12-week postoperative period and, once euthanized and examined, both dehiscence of the sutures and tissue loss in the area of the median nerve lesion were noted.

\section{Functional analysis}

All rats were submitted to a grasping test for functional evaluation, as classically described (5). For the test, each rat was elevated by the tail and stimulated to grab a metal hook that comprised the apex of a metal pyramidal frame. The total frame weighed $700 \mathrm{~g}$ and rested on a conventional digital scale. The left upper extremity of the rat had been previously immobilized with adhesive tape. To examine finger flexion, we observed the rat grasping the hook while being held. At the same time, a second observer filmed the procedure and recorded the weight registered on the scale at the exact moment that the rat released the hook. The difference between the weight of the metal frame (700 g) and the weight registered by the second observer was used to estimate the force generated by the muscle flexors of the fingers. When there was flexion of the palm or wrist, the data were excluded.
Rats were submitted to the grasp test both preoperatively and postoperatively. After repair of the nerve injury, each rat underwent the grasping test three times every 2 weeks through the 12th week, with the average of each three grouped tests used to represent the mean flexor strength at each time. These values were compared between the three groups to determine the rate and degree of functional recovery. Rats were weighed before each grasp test. At the end of the 12th postoperative week, rats were sacrificed by ip injection of a lethal dose of pentobarbital.

\section{Histological analysis}

Ten rats were chosen at random from each group. The nerve segments proximal and distal to the repair were dissected. A 2-cm segment of the nerve including the repair region was removed. Two 4-mm long segments were obtained, $4 \mathrm{~mm}$ proximal and $4 \mathrm{~mm}$ distal to the repair site, and fixed in a solution of $2 \%$ osmium tetroxide. Transverse sectioning was performed and $1-\mu \mathrm{m}$ sections were obtained and stained with $1 \%$ toluidine blue. For histological analysis, each section was photographed using a digital camera (Coolpix; Nikon, Japan) coupled to a light microscope, the objective of which had a magnification of 40 times. One random field of each proximal and distal segment was photographed. Each picture captured an area of 36,000 $\mu \mathrm{m}^{2}$. The pictures from the proximal and distal segment of each nerve were analyzed using the Sigma Scan Pro 5.0 software (SPSS Inc., USA). We obtained the total number and diameter of myelinated fibers. Two indices, expressed as a percentage, were calculated from these parameters, namely, the regeneration index and the diameter change index. The regeneration index was calculated by dividing the total number of regenerated axons in the distal segment by the total number of regenerated axons in the proximal segment, resulting in the percentage of axons that crossed the repair site. The diameter change index was calculated by dividing the mean diameter of regenerated axons in the distal segment by the mean diameter of regenerated axons in the proximal segment (6). These indexes were compared to the results obtained in the functional evaluations to determine the extent to which functional recovery reflected the histological findings (6).

\section{Statistical analysis}

Data are reported as means \pm standard deviation. For the functional test, data were analyzed by two-way repeated measures analysis of variance (ANOVA) followed by the Tukey post hoc test. For histological analysis, oneway ANOVA was used with the level of significance set at $P<0.05$.

\section{Results}

\section{Functional analysis}

Weight gain was observed in all rats throughout the 12 
weeks, and therefore it was not necessary to exclude any animal due to weight loss.

Figure 1 illustrates the median nerve profiles of the three study groups obtained at the time of the first evaluation (t0) and at the end of the 6th, 8th, 10th, and 12th weeks after surgery. After surgery, all groups ultimately displayed evidence of increasing median nerve function over time. Statistically significant differences were apparent, however, at week $8(P=0.019)$ and week $12(P<0.001)$. Post hoc analysis of week 8 data revealed greater grasping strength in group SFM than in group $S(P=0.05)$. At week 12 , the grasping strength of group SFM was greater than that of both group $S(P<0.001)$ and group $S F(P=0.001)$ and, at the same time, the strength of group SF was greater than that of group $S(P=0.014)$. At week 12 , groups $S F$ and $S$ failed to achieve their respective average values estimated before surgery (t0). The SFM group showed total functional recovery.

\section{Histological analysis for axonal regeneration}

Table 1 presents descriptive statistics for the three study groups. No difference was observed between the parameters evaluated. The requirement for similar variances between groups was met, justifying the use of ANOVA.

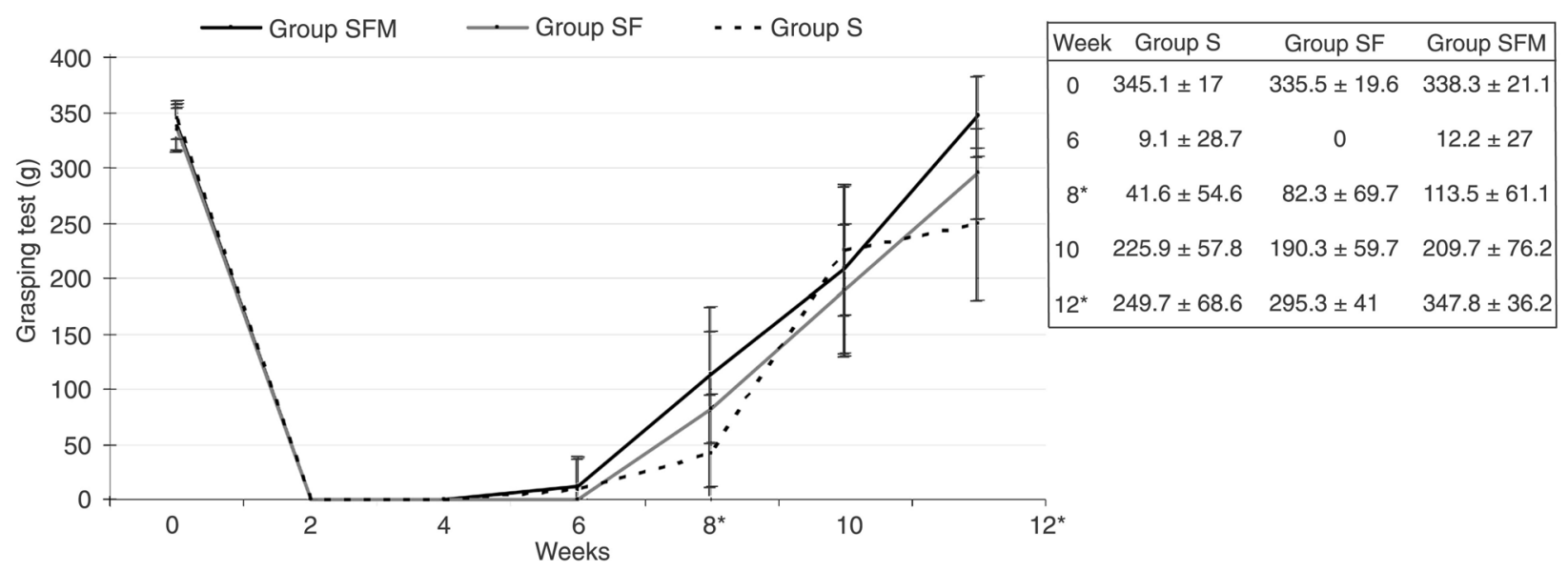

Figure 1. Median nerve profiles during the grasping test in the three study groups. Data are reported as means \pm standard deviation. Group S = epineural neurorrhaphy; group SF = epineural neurorrhaphy plus tissue fibrin adhesive; group SFM = epineural neurorrhaphy plus tissue fibrin adhesive and neurotrophic factor. *At week 8 , grasping strength was greater in group SFM than in group $S$ ( $P$ $=0.05)$. *At week 12, grasping strength was greater in group SFM than in both group $S(P<0.001)$ and group $S F(P=0.001)$. Data were analyzed by two-way repeated measures ANOVA followed by the post hoc Tukey test.

Table 1. Results of histomorphometric evaluation after statistical comparison between the groups.

\begin{tabular}{lccc}
\hline Group parameter & Group S & Group SF & Group SFM \\
\hline PA & $520.20 \pm 89.21$ & $489.30 \pm 59.91$ & $511.00 \pm 227.60$ \\
DA & $849.00 \pm 191.20$ & $724.70 \pm 107.10$ & $757.50 \pm 156.90$ \\
RI $(\%)$ & $164 \pm 27$ & $150 \pm 27$ & $172 \pm 71$ \\
PD $(\mu \mathrm{m})$ & $5.89 \pm 1.24$ & $5.78 \pm 1.24$ & $6.56 \pm 1.45$ \\
DD $(\mu \mathrm{m})$ & $3.57 \pm 0.56$ & $3.77 \pm 0.37$ & $3.96 \pm 0.74$ \\
DCl $(\%)$ & $62 \pm 11$ & $67 \pm 10$ & $67 \pm 40$ \\
\hline
\end{tabular}

Data are reported as means \pm standard deviation. $\mathrm{PA}=$ total number of proximal axons; $\mathrm{DA}=$ total number of distal regenerated axons; $\mathrm{RI}=$ regeneration index; $\mathrm{PD}=$ diameter of regenerated axons in the proximal segment; $D D=$ diameter of regenerated axons in the distal segment; $\mathrm{DCI}=$ diameter change index; group $\mathrm{S}=$ epineural neurorrhaphy; group SF = epineural neurorrhaphy plus tissue fibrin adhesive; group SFM = epineural neurorrhaphy plus tissue fibrin adhesive and neurotrophic factor. No significant difference was observed between groups (two-way ANOVA). 


\section{Discussion}

Group SFM was functionally superior to the other two groups at weeks 8 and 12 and showed total functional recovery over 12 weeks, although histological analysis failed to reveal any significant difference between the three groups. Fukuyama et al. (3) compared intrafascicular injection of $50 \mu \mathrm{L}$ Ringer solution (Lactec ${ }^{\circledR}$ injection, Ohtsuka, Japan) with or without MDP at $2 \mathrm{mg} / \mathrm{mL}$ into the crushed sciatic nerve of rats. The injection was done with a syringe attached with an ultrafine needle (29G) slightly distal to the crushed site. In the MDP group, they reported an increase in the axonal elongation measured by the pinch test 5 days after the crush operation $(P<0.05)$ and increased functional recovery measured by the sciatic nerve functional index in walking track analysis, 3 weeks after the operation and injection $(P<0.01)(3)$.

In our study, histological analysis failed to confirm any immunoadjuvant activity of MDP. The discrepancy between the results of the functional and histological analysis might be explained by the grasping test being able to assess viable nerve fibers among those still regenerating. Moreover, regenerating axons might undergo misdirected growth leading to aberrant muscle reinnervation $(7,8)$ and the presence of polyneuronally innervated muscle fibers may reduce the efficiency of peripheral nerve regeneration (9). The group with the worst results was the suture alone group (S), especially compared to the MDP-treated group (SFM) at weeks 8 and 12. As some investigators have speculated, neurorrhaphy could hinder nerve regeneration in several ways: by escalating the inflammatory response at the repair point, by causing deviations in the path of those axons otherwise aiming towards the distal segment of the nerve (10), by temporarily reducing blood flow through the microcirculation (11), and via the formation of mechanical obstructions such as scar tissue.

The use of neurotrophic substances has been of special interest to those investigating ways by which to optimize nerve regeneration. MDP is a minimum structural unit responsible for the immunoadjuvant activities of bacterial cell wall peptidoglycans in a variety of species (4). It is known that MDP can be considered a functional lipopolysaccharide (LPS) analogue (12). MDP is an immuno-adjuvant that promotes increased activity among microglial phagocytes, Schwann cells and macrophages (2). Wallerian degeneration involves cellular and molecular activities coordinated by a complex network of cytokines that also contribute to macrophage recruitment and activation and, consequently, to macrophage-dependent functions like myelin removal by phagocytosis (13). Adecreased response of these cells in injured nerves has been associated with decreased axonal regeneration (14). However, an interven- tion can accelerate the regenerative process by introducing inflammatory cells or an enhanced inflammatory response (14). The macrophages rapidly remove myelin debris that contain neurite growth inhibitors like myelin-associated glycoprotein. They release cytokines such as interleukin -1 (IL-1) and macrophage inflammatory protein-1 (MIP-1), which probably stimulate Schwann cells and other types in the nerves (3) and growth factors that facilitate axonal regeneration (14). One of the activation mechanisms of macrophages occurs through tumoral necrosis factor-alpha (TNF- $\alpha$ ), which is one of the first cytokines synthesized by Schwann cells during the Wallerian degeneration process (15). TNF-a directly stimulates the macrophages (16) and induces the production of granulocyte-macrophage colony-stimulating factor (GM-CSF) by fibroblasts, promoting macrophage activation. MDP is capable of inducing the formation of several cytokines like TNF- $\alpha$ or stimulates cells to produce them (17), indirectly contributing to macrophage activation. Spinal cord cells were induced to proliferate temporarily by the peripheral administration of certain stimulators of the innate immune system, including MDP (18). Toll-like receptor (TLR) and NOD2 ligands are key receptors that allow mammals to detect the presence of infection by recognizing specific microorganism-derived biomolecules, the so-called pathogen-association molecular patterns (PAMPs). MDP is an NOD2 ligand. Recognition of PAMPs through TLR and NOD2 receptors appears to link the inflammatory and regenerative process. However, other studies have shown that myelin fraction-stimulated macrophages release neurotrophic factors that increase the survival and neurite regeneration of adult dorsal root ganglion neurons in culture (19). Adding MDP or LPS to the culture medium did not enhance neurotrophic secretion by macrophages. These observations suggest that myelin may be a specific activator of macrophages in nerve regeneration.

In summary, using a transected median nerve rat model, we demonstrated enhanced functional recovery twice along the postoperative period of 12 weeks in rats treated with the combination of suturing, fibrin tissue adhesive and the neurotrophic factor MDP compared with rats treated either with neurorrhaphy alone or with sutures plus FTA without MDP. Histological analysis, however, did not identify any difference by which to explain the enhanced functional recovery.

\section{Acknowledgments}

Research supported by FAPESP. The neurotrophic factor MDP was supplied by the Department of Cellular Biology and Development, Institute of Biomedical Sciences, USP, São Paulo, SP, Brazil. 


\section{References}

1. Bruck W. The role of macrophages in Wallerian degeneration. Brain Pathol 1997; 7: 741-752.

2. Parant MA, Parant FJ, Le Contel C, Lefrancier P, Chedid L. MDP derivatives and resistance to bacterial infections in mice. Adv Exp Med Biol 1992; 319: 175-184.

3. Fukuyama R, Takeda H, Fushiki S, Yamamoto T. Muramyl dipeptide injected into crushed sciatic nerve, activates macrophages and promotes recovery of walking locomotion in rats. Restor Neurol Neurosci 1998; 13: 213-219.

4. Inoe AP, Pereira FC, Stopiglia AJ, Da-Silva CF. Pharmacological immunomodulation enhances peripheral nerve regeneration. Pesq Vet Bras 2007; 27: 363-369 (http://www. scielo.br/pdf/pvb/v27n9/v27n9a02.pdf).

5. Bertelli JA, Mira JC. The grasping test: a simple behavioral method for objective quantitative assessment of peripheral nerve regeneration in the rat. $J$ Neurosci Methods 1995; 58: 151-155.

6. Martins RS, Siqueira MG, Da Silva CF, Plese JP. Overall assessment of regeneration in peripheral nerve lesion repair using fibrin glue, suture, or a combination of the 2 techniques in a rat model. Which is the ideal choice? Surg Neurol 2005; 64 (Suppl 1): S1-S6.

7. Dellon AL, Mackinnon SE. Sciatic nerve regeneration in the rat. Validity of walking track assessment in the presence of chronic contractures. Microsurgery 1989; 10: 220-225.

8. Bodine-Fowler SC, Meyer RS, Moskovitz A, Abrams R, Botte MJ. Inaccurate projection of rat soleus motoneurons: a comparison of nerve repair techniques. Muscle Nerve 1997; 20: 29-37.

9. Benfrech E, Alnot JY, Henin D. [An experimental study of nerve sutures and grafts of the sciatic nerve in the rat using fibrin glue]. Ann Chir Main 1989; 8: 296-299.
10. Smahel J, Meyer VE, Bachem U. Glueing of peripheral nerves with fibrin: experimental studies. $J$ Reconstr Microsurg 1987; 3: 211-220.

11. Moy OJ, Peimer CA, Koniuch MP, Howard C, Zielezny Katikaneni PR. Fibrin seal adhesive versus nonabsorbable microsuture in peripheral nerve repair. $J$ Hand Surg Am 1988; 13: 273-278.

12. Hoffmann JA, Kafatos FC, Janeway CA, Ezekowitz RA. Phylogenetic perspectives in innate immunity. Science 1999; 284: 1313-1318.

13. Ramon y Cajal S. Degeneration and regeneration of the nervous system. London: Oxford University Press; 1928.

14. Kiefer R, Kieseier BC, Stoll G, Hartung HP. The role of macrophages in immune-mediated damage to the peripheral nervous system. Prog Neurobiol 2001; 64: 109-127.

15. Shamash S, Reichert F, Rotshenker S. The cytokine network of Wallerian degeneration: tumor necrosis factor-alpha, interleukin-1alpha, and interleukin-1beta. J Neurosci 2002; 22: 3052-3060.

16. Moonis M, Ahmad I, Bachhawat BK. Macrophages in host defence - an overview. Indian J Biochem Biophys 1992; 29: 115-122.

17. Wolfert MA, Murray TF, Boons GJ, Moore JN. The origin of the synergistic effect of muramyl dipeptide with endotoxin and peptidoglycan. J Biol Chem 2002; 277: 39179-39186.

18. Su Y, Zhang Z, Trautmann K, Xu S, Schluesener HJ. TLR and NOD2 ligands induce cell proliferation in the rat intact spinal cord. J Neuropathol Exp Neurol 2005; 64: 991-997.

19. Hikawa N, Takenaka T. Myelin-stimulated macrophages release neurotrophic factors for adult dorsal root ganglion neurons in culture. Cell Mol Neurobiol 1996; 16: 517-528. 